\title{
The Relationship Between Alzheimer's Disease and Skin Diseases: A Review
}

\author{
Hanlin Zhang* \\ Dingyue Zhang* \\ Keyun Tang* \\ Qiuning Sun (1D)
}

Department of Dermatology, State Key Laboratory of Complex Severe and Rare Diseases, National Clinical Research Center for Skin and Immune Diseases, Peking Union Medical College Hospital, Chinese Academy of Medical Sciences and Peking Union Medical College, Beijing, 100730, People's Republic of China

*These authors contributed equally to this work
Correspondence: Qiuning Sun Tel/Fax +86-10-691561/4

Email doctorjennyl@163.com

\begin{abstract}
Alzheimer's disease is the most common type of dementia placing a heavy burden on the healthcare system worldwide. Skin diseases are also one of the most common health problems. Several skin diseases are associated with Alzheimer's disease through different mechanisms. This review summarizes the relationship between Alzheimer's disease and several types of skin diseases, including bullous pemphigoid, hidradenitis suppurativa, psoriasis, skin cancer, and cutaneous amyloidosis, and provides suggestions based on these associations. Neurologists, dermatologists, and general practitioners should be aware of the relationship between Alzheimer's disease and skin diseases. Dermatology/neurology consultation or referral is necessary when needed.
\end{abstract}

Keywords: Alzheimer's disease, dementia, skin disease, bullous pemphigoid, hidradenitis suppurativa, psoriasis

\section{Introduction}

Dementia is a neurological disorder defined by progressive cognitive function impairment and has a growing incidence in the aged population. Around 50 million patients are suffering from dementia worldwide, which places a huge burden on the healthcare system. The pathogenesis of dementia remains unclear, and many risk factors have been identified. ${ }^{1}$ Among all the subtypes of dementia, Alzheimer's disease is the most common one, accounting for $43.5 \%$ of all cases. ${ }^{2}$ The neuropathological feature of Alzheimer's disease is the accumulation of pathological amyloid- $\beta$ and tau in the brain. According to family history, Alzheimer's disease can be divided into familial Alzheimer's disease and sporadic Alzheimer's disease, and multiple risk factors have been identified in both of them. ${ }^{3}$

Skin diseases are also one of the most common health disorders. Some studies have noted the relationship between Alzheimer's disease and skin diseases. In patients with Alzheimer's disease, the physiology of skin was altered, ${ }^{4}$ and neurodegenerative disease-related proteins were detectable within human skin. ${ }^{5}$ For patients with Alzheimer's disease, the risk of developing bullous pemphigoid was elevated by 2.6 times. $^{6}$ In 2013, a study found that nonmelanoma skin cancer was associated with a significantly reduced the risk of Alzheimer's disease. ${ }^{7}$

Although there is evidence that several skin diseases are associated with Alzheimer's disease, no review summarized their association. This review aims to summarize the relationship between Alzheimer's disease and several types of skin diseases, including bullous pemphigoid, hidradenitis suppurativa, psoriasis, skin cancer, and cutaneous amyloidosis, and provide suggestions based on these 
associations, which may give dermatologists and neurologists a novel perspective on the relationship between Alzheimer's disease and skin diseases. This review did not involve intervention or data collection in animal experiments or clinical trials. Approval from an ethical committee was not needed.

\section{Alzheimer's Disease and Bullous Pemphigoid}

Bullous pemphigoid (BP) is an autoimmune skin disease, characterized by limited or diffuse tense subepidermal bullae on urticarial, erythematous bases. ${ }^{8-12}$ This disease occurs mostly in the elderly, with an incidence rate of 12 66 new cases per 1 million people per year. ${ }^{13}$

Many comorbidities have been identified in BP patients, such as cardiovascular diseases, diabetes mellitus, neurological diseases, psychiatric diseases, and malignancies, and the strongest association has been found between $\mathrm{BP}$ and neurological diseases. ${ }^{14} \mathrm{~A}$ recent meta-analysis including twelve case-control and two cohort studies revealed that BP was associated with an increased risk for dementia $(R R=4.46)$, stroke $(R R=2.68)$, epilepsy $(\mathrm{RR}=2.98)$, and multiple sclerosis $\quad(\mathrm{RR}=12.40) .{ }^{15}$ Meanwhile, existing neurological diseases also increased the risk for BP. ${ }^{6}$ For most patients, at least one neurological disorder was diagnosed prior to BP, with a median duration of 5.5 years. ${ }^{16}$ In 2019, a study evaluated demographic features and autoantibody levels in $77 \mathrm{BP}$ control patients and $33 \mathrm{BP}$ patients with preceding neurological disorders. They found that patients with BP who had preceding neurological diseases had a shorter elapsed time between the onset of skin disease and BP diagnosis. No significant differences in clinical presentation, BP severity scores, or autoantibody responses were observed among the groups. The clinical phenotype of BP was not affected by preceding neurological diseases despite the age difference. ${ }^{17}$

Several studies have confirmed the association between dementia and BP with a risk ratio ranging from 2.2 to $4.8,{ }^{14,18-20}$ but Alzheimer's disease has rarely been studied as an isolated subtype of dementia due to the nature of retrospective studies. In 2016, a nationwide Finnish study first revealed an elevated RR of 2.6 in patients with Alzheimer's disease, and in those with vascular dementia, the value was 3.6. ${ }^{6}$ This result was in accordance with the outcome $(\mathrm{OR}=2.6)$ of a population-based matched-cohort study with 3,281 BP patients in 2017. ${ }^{21}$ A multi-centered case-control study evaluated the cognitive function using Mini-Mental State Examination (MMSE) and Montreal Cognitive Assessment (MoCA) scores and found decreased cognitive abilities and a higher risk of cognitive impairment in BP patients, which showed that these patients were more likely to develop dementia. ${ }^{12}$ However, in a single-centered cohort study with 183 BP patients, Alzheimer's disease was the only neurological disorder with no significant association with $\mathrm{BP}{ }^{22}$

BP is characterized by the self-produced IgG against two autoantigens: BP180 (BPAG2) and BP230 (BPAG1e), both of which act as epidermal adhesion molecules, expressing not only in the skin but also widely in brain neurons. ${ }^{23,24}$ The presence of proteins shared between the central nervous system and the skin which are recognized at autoantigens in the central nervous system and the epidemiological data between BP and neurological diseases lead to the hypothesis that neurodegeneration or neuroinflammation may disrupt the blood-brain barrier, causing a cross-reactive immune response between skin and brain autoantigens and thus resulting in $\mathrm{BP}^{25}$ This hypothesis has also been further supported by anatomical and autoimmunological evidence. BP180 has been found mainly in pyramidal cells from the ganglionic layer of the cortex and hippocampus, which is also the common lesion site of Alzheimer's disease. ${ }^{26}$ A study in 2011 first examined the serum samples by immunoblotting against BP230 and BP180 extracted of both human skin and brain. They found that a larger proportion of patients with both BP and neurological diseases have sera that could recognize BP230 than those from BP group or control group, indicating the potential existence of cross-immunity. ${ }^{27}$ The presence of circulating BP antibodies has been further investigated by ELISA in patients with different neurological diseases, ${ }^{28-30}$ and the association between diagnosis of dementia and the existence of BP180 antibody in blood has been verified. A study including 115 Alzheimer's disease patients and 40 controls found BP $180 \mathrm{lgG}$ autoantibodies in $17 \%$ patients with Alzheimer's disease, but only $3 \%$ in control groups. The same tendency existed in BP230 but not significantly. ${ }^{28}$ This study also reported a negative correlation between cognitive function and the level of BP180 antibody. Similar results were reported in multiple sclerosis and Parkinson patients. ${ }^{29,30}$

There is also growing evidence against this hypothesis. In contrast with former studies, a recent study failed to find BP180 in the hippocampus of the human brain, ${ }^{31}$ and this finding was also supported by The Human Protein 
Atlas. ${ }^{32}$ The serum of Alzheimer's disease and Parkinson's disease patients that could recognize the full-length BP180 in Western blot could not bind the cutaneous basement membrane in indirect immunofluorescence analysis. Moreover, none of these patients showed BP-like symptoms. ${ }^{28,29}$ A recent study also showed that in Alzheimer's disease and multiple sclerosis patients, the BP180 autoantibodies targeted the intracellular and midextracellular parts. However, in BP patients, it targeted the well-known non-collagenous 16A domain, indicating a different epitope profile and the insufficiency of the existing cross-reactive immunology hypothesis. ${ }^{33}$ Another study proved that HLA-DQB1*03:01, which was overexpressed in BP patients, might increase the T-cell avidity to BP180, leading to genetic susceptibility to developing BP upon exposure to target antigens in neurons of neurocognitive patients. ${ }^{34}$ This provided us a new perspective on the relationship between neurological and cutaneous disorders.

\section{Alzheimer's Disease and Hidradenitis Suppurativa}

Hidradenitis suppurativa (HS, also known as acne inversa) is a chronic, painful skin disease characterized by recurrent painful nodules, abscesses, and tunnels of intertriginous sites. ${ }^{35-38}$ It occurs mostly between the second and fourth decades, affecting $1 \%$ of the adult European population with a huge impact on their quality of life. ${ }^{39}$ To date, the etiology of HS remains unclear, with studies basically on cytokines (TNF $\alpha$ and IL-17), hormones, genetics, and microbiology. ${ }^{40}$

Alzheimer's disease and HS are considered related due to genetic factors. Familial HS and familial Alzheimer's disease share common mutations in $\gamma$-secretase genes. $\gamma$ secretase complex is a transmembrane protease targeting more than 60 substrates, most markedly the amyloid precursor protein (APP) and Notch signal pathway. The complex is composed of four subunits encoded by six genes: presenilin (PSEN1/PSEN2), presenilin enhancer-2 (PSENEN), nicastrin (NCSTN), and anterior pharynx defective (APH1A/APH1B). PSEN1 and PSEN2 mutations have been identified in familial Alzheimer's disease, leading to deficient cleaving of APPs and accumulation of amyloid plaques. ${ }^{41}$

Heterozygous $\gamma$-secretase gene mutations in PSENEN, NCSTN, PSEN1 have been reported in HS families with an autosomal dominant inheritance and incomplete penetrance, whereas the mechanism remains unclear. ${ }^{42}$ Deficiency of $\gamma$-secretase complex caused by its inhibitor Semagacestat led to cutaneous side effects, such as skin rashes, hair color changes, and increased skin cancer risks. ${ }^{43}$ In mouse models, deficiency in PSEN1, PSEN2, or NCT caused HS symptoms: absent sebaceous gland, formation of epidermal cysts, follicular keratinization and atrophy; some of these mice even had squamous cell carcinoma. ${ }^{44-46}$ Notch knock-out mice also showed HStrait, indicating the involvement of Notch-signaling pathway in HS. ${ }^{45}$ Amyloidosis is a key step in Alzheimer's disease pathology. In 2012, a case report reported that an HS patient developed systemic amyloid A amyloidosis, suggesting a possible link between Alzheimer's disease and $\mathrm{HS}^{47}$

However, more recent studies do not support the association between the two diseases. Evidence is growing that phenotypic heterogeneity in HS spectrum divides HS into several categories. ${ }^{48} \gamma$-secretase mutated patients, with more severe, widespread, and treatment-resistant symptoms, are considered only one of its subtypes. ${ }^{49}$ The correlation in genetics is also not as strong as expected. Though many studies are focused on familial HS, case series evidence indicated that only one-third of HS patients reported an HS family history. ${ }^{48}$ Only a minority of patients were detected $\gamma$-secretase mutations even in those with a family history. ${ }^{50,51}$ While most reported HS mutations occurred on NCSTN, only one PSEN1 (the mutated gene in familial Alzheimer's disease) was found without any Alzheimer's disease-like symptoms in the patient. Case reports of several members in one HS family also suggest $\gamma$-secretase mutation cannot produce the HS phenotype by itself. $^{52}$ In 2017, two large cohort studies with 3,432 and 28,755 HS patients, respectively, both demonstrated no increased risk for Alzheimer's disease, with the ratio slightly and insignificantly increased $(\mathrm{HR}=1.44, \quad 0.79-2.64 ; \quad \mathrm{OR}=1.23, \quad 0.96-1.56$, respectively) ${ }^{53,54} \mathrm{~A}$ systematic review ${ }^{55}$ and an in silico analysis summarized the existing HS mutation cases ${ }^{56}$ and demonstrated no mutation overlap between the two diseases. The substrate recognition and cleavage of $\gamma$ secretase between familial HS and Alzheimer's disease are also different. ${ }^{57}$ The only statistical evidence that supports the correlation is a cross-sectional analysis based on family history in 2020, which demonstrated a positive correlation between the family history of HS and the family history of Alzheimer's disease among 192 HS patients, and a 4.5 times increased risk of family history 
of Alzheimer's disease among familial HS patients was observed. $^{58}$

\section{Alzheimer's Disease and Psoriasis}

Psoriasis is an immune-mediated inflammatory skin disease characterized by chronic plaques covered by silverywhite scales. ${ }^{59,60}$ This disease affects $0.5-11.4 \%$ adults, causing huge negative effects on their quality of life. ${ }^{61-63}$ Nowadays, psoriasis is considered a systematic condition associated with many comorbidities: cardiovascular comorbidities, other immune-mediated diseases, and mental disorders. Psoriasis and dementia are both inflammatory disorders, and their link has been investigated.

Previous studies have demonstrated an increased risk for psoriasis patients to develop dementia with a risk ratio ranging from $1.10-1.25 .^{64-67}$ The risk of developing psoriasis also increases among dementia patients. ${ }^{68}$ However, one study in 2018 found psoriasis had a surprisingly protective function for dementia $(\mathrm{HR}=0.54) .{ }^{69}$ According to a recent meta-analysis, the risk of developing vascular dementia and non-vascular dementia in psoriasis patients was higher than patients without psoriasis $(R R=1.41$, $R R=1.13$, respectively). ${ }^{70}$ Among those with severe psoriasis, the risk of death from dementia rose over three times $(\mathrm{HR}=3.64)$, which meant dementia was one of the major causes of death for these patients. ${ }^{71}$ A population-based case-control cohort study in Korea investigated the link between psoriasis and Alzheimer's disease, and found a slightly but significantly increased risk of Alzheimer's disease in psoriasis patients than those without psoriasis $(\mathrm{HR}=1.09) .{ }^{66}$ The association was found significantly stronger in middle-aged patients than elderly patients ( $H R=1.30, H R=1.08$, respectively). Some cross-sectional studies investigated the pre-clinical markers for dementia: two studies revealed that psoriasis patients had a higher risk of mild cognitive impairment, especially in visuospatial function, verbal memory, and executive function; ${ }^{72,73}$ while one study demonstrated no association. ${ }^{69}$ Despite a non-significantly decreased hippocampal volume and larger white matter lesions, MRI markers showed no change in psoriasis patients. ${ }^{69}$

The mechanisms for psoriasis and Alzheimer's disease are complicated and remain unclear, but the epidemiological association suggests a shared mechanism of disease pathogenesis or genetic susceptibility. Psoriasis is considered an inflammatory and immune disease. In psoriasis lesions, activated $\mathrm{T}$ cells and dendritic cells are accumulated, producing cytokines such as TNF $\alpha$ and IL23, and these cytokines will further migrate to the epidermis and act on keratinocytes. As a key pro-inflammatory factor, TNF- $\alpha$ also plays a vital part in the pathogenesis of Alzheimer's disease, exacerbating $A \beta$ and tau pathologies in vivo. ${ }^{74}$ TNF blocking agents can improve the cognitive function in patients with Alzheimer's disease, ${ }^{74}$ and a case-control study proved that psoriasis patients treated with TNF blocking agents such as etanercept, adalimumab, and infliximab have a lower risk for developing Alzheimer's disease than those without such treatment $(\mathrm{OR}=0.47){ }^{75} \mathrm{IL}-12 / 23$ axis is important in psoriasis development, and monoclonal antibodies targeting IL-12/ IL-23 common subunit p40 are widely used as a drug to treat psoriasis. $^{76}$ Recent reviews have also verified the important roles of IL-23/IL-12A axis in the pathogenesis of age-associated inflammation in Alzheimer's disease. ${ }^{77,78}$ For the mouse model of Alzheimer's disease, p40 level in cerebrospinal fluid was increased, and the blockage of $\mathrm{p} 40$ led to fewer $\mathrm{A} \beta$ plaques and improved cognitive deficits. ${ }^{79}$

There is also genetic evidence supporting the link between psoriasis and Alzheimer's disease. Apolipoprotein E (APOE) is the strongest genetic risk factor for Alzheimer's disease. As the main cholesterol carrier, it greatly affects $A \beta$ deposition and tau phosphorylation and is also related to cardiovascular diseases and other neurodegenerative disorders. ${ }^{80}$ Similarly, many studies have confirmed that APOE genotypes can be an independent risk factor for the onset and severity of psoriasis. $^{81-84}$ A meta-analysis including seven studies with 966 psoriasis patients and 1,086 controls revealed that people with $\varepsilon 3$ allele or $\varepsilon 3 / \varepsilon 3$ genotype had a decreased possibility to develop psoriasis, whereas $\varepsilon 2$ allele could increase the risk. ${ }^{83}$ In 2016, a study analyzed the genetic overlap between Alzheimer's disease and immune-mediated diseases. Eight polymorphisms and two pleiotropic loci were found associated with Alzheimer's disease and the six diseases, including psoriasis. This result suggests that inflammation may be related to the onset of Alzheimer's disease. ${ }^{85}$

Given these shared mechanisms, the benefit of treating psoriasis as a systemic disease has been emphasized. A cohort study demonstrated that psoriasis patients who received systemic anti-inflammatory therapy for at least three months were less likely to develop dementia. ${ }^{67}$ A Korean study also divided psoriasis patients into systemic therapy group and non-systemic therapy group. For those who received systemic therapy, the risk of 
developing Alzheimer's disease was significantly lower than in those in no systemic therapy group, and even lower than in those without psoriasis (no systemic therapy: 6.48; systemic therapy: 3.70; controls without psoriasis: 5.59 (per 1,000 person-years)). ${ }^{66}$

\section{Alzheimer's Disease and Skin Cancer}

Epidemiologic studies have demonstrated that patients with neurodegenerative disorders have lower malignancy rates, such as prostate cancer, breast cancer, and nonmelanoma skin cancer. ${ }^{86}$ In 2020, a meta-analysis including $9,630,435$ individuals was conducted to investigate the association between Alzheimer's disease and cancer, and concluded that the risk for people with a cancer history to develop Alzheimer's disease was weakly but significantly decreased $(\mathrm{OR}=0.75$ from case-control studies, $\mathrm{HR}=0.89$ from cohort studies). ${ }^{86}$ Another prospective cohort study proved that such a relationship between Alzheimer's disease and cancer was bidirectional and also observed in Parkinson's disease and Huntington's diseases. ${ }^{87}$

Skin cancer can be divided into nonmelanoma skin cancer (NMSC) and malignant melanoma. ${ }^{88}$ A populationbased longitudinal study with 1,102 adults reported a reduced risk of Alzheimer's disease $(\mathrm{HR}=0.50)$ in NMSC patients compared to those free of NMSC. ${ }^{7}$ Such protective effect was eliminated when considering allcause dementia. Another study demonstrated the same but attenuated trend, with $2-10 \%$ reduced risk of Alzheimer's disease in NMSC patients. ${ }^{89}$ In 2018, a large, single-centered study evaluating the registry data of a Midwestern US population was conducted. A significantly decreased risk of subsequent Alzheimer's disease in both malignant melanoma patients and NMSC patients was also observed. ${ }^{90}$

Many types of cancer and reduced risk of Alzheimer's disease are linked through shared genes and biological pathways. There is a gene overlap between Alzheimer's disease and cancer, with many genes involved in signaling, metabolism, and cell growth. ${ }^{91}$ For example, p53, a tumor suppressor gene, was expressed lower in cancer but upregulated in Alzheimer's disease and other neurodegenerative disorders. ${ }^{92}$ Pin 1 is an enzyme involved in cell cycle control and has an elevated expression level in tumors. Studies found that PIN1 can suppress tau and therefore prevent amyloid- $\beta$ deposition, ${ }^{93}$ and its expression was decreased in the brains of Alzheimer's disease patients. ${ }^{94}$
MicroRNA-455-3p, which was relevant to different types of cancers, could modulate amyloid- $\beta$ protein precursor $(\mathrm{A} \beta \mathrm{PP})$ and amyloid- $\beta$ (A $\beta)$ levels, therefore acted as a biomarker in Alzheimer's disease. ${ }^{95}$

Mechanisms of the link between skin cancer and Alzheimer's disease were also demonstrated. The dysregulation in amyloid precursor protein (APP) processing is an event related to both Alzheimer's disease and cancer. The amyloidogenic pathway is increased in Alzheimer's disease, while the non-amyloidogenic pathway is increased in cancer. ${ }^{96}$ Reducing the expression of APP could impair the proliferation of metastatic melanoma cells and increase their sensitivity to chemotherapeutic drugs. ${ }^{97}$ A recent study found that Yes-associated protein could sense the extracellular accumulation of amyloidogenic proteins and modulate transcriptional activity of proliferative genes, therefore affected melanoma progression. $^{98} \gamma$-secretase inhibitor could attenuate amyloid burden in patients with Alzheimer's disease. However, Alzheimer's disease patients and animal models treated with semagacestat were more likely to develop HSlike symptoms and melanoma, indicating a shared mechanism involving $\gamma$-secretase or Notch-1 pathway among HS, melanoma, and Alzheimer's disease. ${ }^{99,100}$ A recent study also revealed that APOE genotype, which had pleiotropic organismal effects and influenced the risk of Alzheimer's disease, could affect the outcomes in melanoma by modulating anti-tumor immunity. ${ }^{101}$ In contrast to its protective effect on Alzheimer's disease, APOE2 variants indicated poor outcomes in melanoma patients, while APOE4, an important risk factor in Alzheimer's disease, indicated better outcomes.

Confounders, such as education, income, lifestyle, physical activity, may also play a role in the correlation. For example, more physical activities could reduce the risk for cognitive decline and dementia, ${ }^{102}$ whereas the increased exposure to $\mathrm{UV}$ radiation in outdoor activities might increase the skin cancer risk. ${ }^{103}$ A cohort study found that the baseline cognitive function was better in NMSC patients, ${ }^{7}$ which was consistent with previous studies that NMSC was found associated with higher income and education. $^{104}$ Clinicians may also be less likely to diagnose Alzheimer's disease in patients with prior malignancy. However, a recent meta-analysis evaluated the possible bias and found that the inverse association existed, partly due to shared etiological mechanism or survival bias, but could not be explained by confounders 
such as diagnostic bias, competing risks bias, or inappropriate control. $^{86}$

\section{Alzheimer's Disease and Cutaneous Amyloidosis}

Amyloidosis might occur locally both in brain and skin, leading to neurodegenerative and skin conditions, respectively. Studies indicated that there might be a connection between the amyloid deposits in Alzheimer's disease and cutaneous amyloidosis. ${ }^{4,105,106}$ The pathology for skin and brain amyloidosis might be similar, and their amyloid deposits shared a common ultrastructure. Presenilin-1 and ApoE4, which were involved in Alzheimer's disease, also played a role in the amyloidogenesis in the skin. In 2018, a patient with small, brownish macules and severe itching on the face, upper back, and abdomen was diagnosed with both Alzheimer's disease and macular amyloidosis. ${ }^{107}$ However, more studies are needed to prove the skinbrain axis. $^{108}$

\section{Conclusions}

This review summarizes the relationship between Alzheimer's disease and bullous pemphigoid, hidradenitis suppurativa, psoriasis, skin cancer, and cutaneous amyloidosis, and provides suggestions based on these associations. Generally, bullous pemphigoid and psoriasis may increase the risk of Alzheimer's disease and vice versa, while skin cancer may have a protective effect on Alzheimer's disease and vice versa. The relationship between hidradenitis suppurativa, cutaneous amyloidosis, and $\mathrm{AD}$, however, is still unclear.

Although we still cannot say that there exists a correlation between Alzheimer's disease and skin diseases based on current evidence, this review may have some clinical applications. Neurologists, dermatologists, and general practitioners should be aware of the relationship between Alzheimer's disease and skin diseases. If neurologists or general practitioners observe related skin symptoms in patients with Alzheimer's disease, dermatology consultation or referral is needed. When dermatologists or general practitioners receive patients with these skin diseases, attention should also be paid to whether they have AD signs or symptoms. Neurology consultation or referral is needed if necessary.

This review has several limitations. First, only five selected skin diseases were discussed. Authors retrieved relevant articles in several databases, and identified the five most-discussed diseases. Many other skin diseases may also be associated with Alzheimer's disease but are not summarized in this review. Second, mechanisms of the association between Alzheimer's disease and skin diseases have not been well illustrated. Further studies should concentrate on the underlying mechanisms of the correlation between Alzheimer's disease and skin diseases. Larger, prospective studies with longer follow-up are warranted. All these contributions rely much on the close cooperation among dermatologists, neurologists, and neuroscientists. ${ }^{109}$

\section{Disclosure}

The authors report no conflicts of interest in this work.

\section{References}

1. Zhang H, Chen K, Wang N, et al. Analysis of brain donors' demographic and medical characteristics to facilitate the construction of a human brain bank in China. $J$ Alzheimers Dis. 2018;66 (3):1245-1254. doi:10.3233/JAD-180779

2. Goodman RA, Lochner KA, Thambisetty M, Wingo TS, Posner SF, Ling SM. Prevalence of dementia subtypes in United States Medicare fee-for-service beneficiaries, 2011-2013. Alzheimers Dement. 2017;13(1):28-37. doi:10.1016/j. jalz.2016.04.002

3. Scheltens P, De Strooper B, Kivipelto M, et al. Alzheimer's disease. Lancet. 2021;397(10284):1577-1590. doi:10.1016/ S0140-6736(20)32205-4

4. Clos AL, Kayed R, Lasagna-Reeves CA. Association of skin with the pathogenesis and treatment of neurodegenerative amyloidosis. Front Neurol. 2012;3:5. doi:10.3389/fneur.2012.00005

5. Akerman SC, Hossain S, Shobo A, et al. Neurodegenerative disease-related proteins within the epidermal layer of the human skin. J Alzheimers Dis. 2019;69(2):463-478. doi:10.3233/JAD181191

6. Försti AK, Jokelainen J, Ansakorpi H, et al. Psychiatric and neurological disorders are associated with bullous pemphigoid a nationwide Finnish Care Register study. Sci Rep. 2016;6:37125. doi:10.1038/srep37125

7. White RS, Lipton RB, Hall CB, Steinerman JR. Nonmelanoma skin cancer is associated with reduced Alzheimer disease risk. Neurology. 2013;80(21):1966-1972. doi:10.1212/WNL.0b013e3182941990

8. Kluger N, Pankakoski A, Panelius J. Depression and anxiety in patients with bullous pemphigoid: impact and management challenges. Clin Cosmet Investig Dermatol. 2020;13:73-76. doi:10.2147/CCID.S212984

9. Delli FS, Sotiriou E, Lazaridou E, et al. Total IgE, eosinophils, and interleukins 16,17A, and 23 correlations in severe bullous pemphigoid and treatment implications. Dermatol Ther. 2020;33 (6):e13958. doi:10.1111/dth.13958

10. Azimi SZ, Firooz A, Murrell DF, Daneshpazhooh M. Treatment concerns for bullous pemphigoid in the COVID-19 pandemic era. Dermatol Ther. 2020;33(6):e13956. doi:10.1111/dth.13956

11. Wang YN, Hammers CM, Mao X, Jin HZ, Yuan J, Li L. Analysis of the autoimmune response against BP180 in patients with Alzheimer's disease. Ann Transl Med. 2021;9(2):107. doi:10.21037/atm-20-5343

12. Zhao W, Wang Y, Mao X, et al. Detection of underlying dementia in bullous pemphigoid patients using cognitive evaluation tests: a multicenter case-control study. Ann Transl Med. 2020;8 (21):1397. doi:10.21037/atm-20-1319 
13. Schmidt E, Zillikens D. Pemphigoid diseases. Lancet. 2013;381 (9863):320-332. doi:10.1016/S0140-6736(12)61140-4

14. Langan SM, Groves RW, West J. The relationship between neurological disease and bullous pemphigoid: a population-based case-control study. J Invest Dermatol. 2011;131(3):631-636. doi: $10.1038 /$ jid.2010.357

15. Lai YC, Yew YW, Lambert WC. Bullous pemphigoid and its association with neurological diseases: a systematic review and meta-analysis. J Eur Acad Dermatol Venereol. 2016;30 (12):2007-2015. doi:10.1111/jdv.13660

16. Taghipour K, Chi CC, Vincent A, Groves RW, Venning V, Wojnarowska F. The association of bullous pemphigoid with cerebrovascular disease and dementia: a case-control study. Arch Dermatol. 2010;146(11):1251-1254. doi:10.1001/archder matol.2010.322

17. Messingham KN, Miller AD, Narayanan NS, Connell SJ, Fairley JA. Demographics and autoantibody profiles of pemphigoid patients with underlying neurologic diseases. $J$ Invest Dermatol. 2019;139(9):1860-1866. doi:10.1016/j.jid.2019.01.034

18. Bastuji-Garin S, Joly P, Lemordant P, et al. Risk factors for bullous pemphigoid in the elderly: a prospective case-control study. J Invest Dermatol. 2011;131(3):637-643. doi:10.1038/ jid.2010.301

19. Chen YJ, Wu CY, Lin MW, et al. Comorbidity profiles among patients with bullous pemphigoid: a nationwide population-based study. Br J Dermatol. 2011;165(3):593-599. doi:10.1111/j.13652133.2011.10386.x

20. Brick KE, Weaver $\mathrm{CH}$, Savica R, et al. A population-based study of the association between bullous pemphigoid and neurologic disorders. $J$ Am Acad Dermatol. 2014;71(6):1191-1197. doi:10.1016/j.jaad.2014.07.052

21. Kibsgaard L, Rasmussen M, Lamberg A, Deleuran M, Olesen $\mathrm{AB}$, Vestergaard $\mathrm{C}$. Increased frequency of multiple sclerosis among patients with bullous pemphigoid: a population-based cohort study on comorbidities anchored around the diagnosis of bullous pemphigoid. Br J Dermatol. 2017;176 (6):1486-1491. doi:10.1111/bjd.15405

22. Papakonstantinou E, Limberg MM, Gehring $M$, et al. Neurological disorders are associated with bullous pemphigoid J Eur Acad Dermatol Venereol. 2019;33(5):925-929. doi:10.1111/ jdv. 15444

23. Seppänen A, Suuronen T, Hofmann SC, Majamaa K, Alafuzoff I. Distribution of collagen XVII in the human brain. Brain Res. 2007;1158:50-56. doi:10.1016/j.brainres.2007.04.073

24. Seppänen A, Autio-Harmainen H, Alafuzoff I, et al. Collagen XVII is expressed in human CNS neurons. Matrix Biol. 2006;25 (3):185-188. doi:10.1016/j.matbio.2005.11.004

25. Kridin K, Hübner F, Recke A, Linder R, Schmidt E. The burden of neurological comorbidities in six autoimmune bullous diseases: a population-based study. J Eur Acad Dermatol Venereol. 2021. doi: $10.1111 / j \mathrm{dv} .17465$

26. Seppänen A. Collagen XVII: a shared antigen in neurodermatological interactions? Clin Dev Immunol. 2013;2013:240570. doi: $10.1155 / 2013 / 240570$

27. Chen J, Li L, Chen J, et al. Sera of elderly bullous pemphigoid patients with associated neurological diseases recognize bullous pemphigoid antigens in the human brain. Gerontology. 2011;57 (3):211-216. doi:10.1159/000315393

28. Kokkonen N, Herukka SK, Huilaja L, et al. Increased levels of the bullous pemphigoid BP180 autoantibody are associated with more severe dementia in Alzheimer's disease. J Invest Dermatol. 2017;137(1):71-76. doi:10.1016/j.jid.2016.09.010

29. Messingham KAN, Aust S, Helfenberger J, et al. Autoantibodies to collagen XVII are present in Parkinson's disease and localize to tyrosine-hydroxylase positive neurons. $J$ Invest Dermatol. 2016;136(3):721-723. doi:10.1016/j.jid.2015.12.005
30. Recke A, Oei A, Hübner F, et al. Parkinson disease and multiple sclerosis are not associated with autoantibodies against structural proteins of the dermal-epidermal junction. $\mathrm{Br} J$ Dermatol. 2016;175(2):407-409. doi:10.1111/bjd.14538

31. Barrick BJ, Ida CM, Laniosz V, et al. Bullous pemphigoid, neurodegenerative disease, and hippocampal BP180 expression: a retrospective postmortem neuropathologic study. J Invest Dermatol. 2016;136(10):2090-2092. doi:10.1016/j.jid.2016 .06 .015

32. Künzli K, Favre B, Chofflon M, Borradori L. One gene but different proteins and diseases: the complexity of dystonin and bullous pemphigoid antigen 1. Exp Dermatol. 2016;25(1):10-16. doi:10.1111/exd.12877

33. Tuusa J, Lindgren O, Tertsunen HM, et al. BP180 autoantibodies target different epitopes in multiple sclerosis or Alzheimer's disease than in bullous pemphigoid. $J$ Invest Dermatol. 2019;139 (2):293-299. doi:10.1016/j.jid.2018.09.010

34. Amber KT, Zikry J, Hertl M. A multi-hit hypothesis of bullous pemphigoid and associated neurological disease: is HLADQB1*03:01, a potential link between immune privileged antigen exposure and epitope spreading? Hla. 2017;89(3):127-134. doi: $10.1111 / \tan .12960$

35. Koumaki D, Efthymiou O, Bozi E, Katoulis AC. Perspectives on perceived stigma and self-stigma in patients with hidradenitis suppurativa. Clin Cosmet Investig Dermatol. 2019;12:785-790. doi:10.2147/CCID.S180036

36. Fernandez JM, Marr KD, Hendricks AJ, et al. Alleviating and exacerbating foods in hidradenitis suppurativa. Dermatol Ther. 2020;33(6):e14246. doi:10.1111/dth. 14246

37. Misitzis A, Goldust M, Jafferany M, Lotti T. Psychiatric comorbidities in patients with hidradenitis suppurativa. Dermatol Ther. 2020;33(4):e13541. doi:10.1111/dth.13541

38. Shah M, Sachdeva M, Melendez-Gonzalez M, Piguet V, Sayed C. Hidradenitis suppurativa and chromosomal abnormalities: a case report and systematic review of the literature. Int $J$ Dermatol. 2021;60(3):261-271. doi:10.1111/ijd.15111

39. Sabat R, Jemec GBE, Matusiak $€$, Kimball AB, Prens E, Wolk K. Hidradenitis suppurativa. Nat Rev Dis Primers. 2020;6(1):18. doi:10.1038/s41572-020-0149-1

40. Nguyen TV, Damiani G, Orenstein LAV, Hamzavi I, Jemec GB. Hidradenitis suppurativa: an update on epidemiology, phenotypes, diagnosis, pathogenesis, comorbidities and quality of life. $J$ Eur Acad Dermatol Venereol. 2021;35(1):50-61. doi:10.1111/ jdv. 16677

41. De Strooper B, Iwatsubo T, Wolfe MS. Presenilins and $\gamma$ secretase: structure, function, and role in Alzheimer Disease. Cold Spring Harb Perspect Med. 2012;2(1):a006304. doi:10.1101/cshperspect.a006304

42. Ingram JR. The genetics of hidradenitis suppurativa. Dermatol Clin. 2016;34(1):23-28. doi:10.1016/j.det.2015.07.002

43. Panza F, Frisardi V, Imbimbo BP, et al. REVIEW: $\gamma$-Secretase inhibitors for the treatment of Alzheimer's disease: the current state. CNS Neurosci Ther. 2010;16(5):272-284. doi:10.1111/ j.1755-5949.2010.00164.x

44. Xia X, Qian S, Soriano S, et al. Loss of presenilin 1 is associated with enhanced beta-catenin signaling and skin tumorigenesis. Proc Natl Acad Sci U S A. 2001;98(19):10863-10868. doi:10.1073/pnas.191284198

45. Pan Y, Lin MH, Tian X, et al. $\gamma$-Secretase functions through notch signaling to maintain skin appendages but is not required for their patterning or initial morphogenesis. Dev Cell. 2004;7(5):731-743. doi:10.1016/j.devcel.2004.09.014

46. Li T, Wen H, Brayton C, et al. Epidermal growth factor receptor and notch pathways participate in the tumor suppressor function of $\gamma$-secretase. J Biol Chem. 2007;282(44):32264-32273. doi:10.1074/jbc.M703649200 
47. Girouard SD, Falk RH, Rennke HG, Merola JF. Hidradenitis suppurativa resulting in systemic amyloid A amyloidosis: a case report and review of the literature. Dermatol Online J. 2012;18 (1):2. doi:10.5070/D30P46V20Q

48. Canoui-Poitrine F, Le Thuaut A, Revuz JE, et al. Identification of three hidradenitis suppurativa phenotypes: latent class analysis of a cross-sectional study. $J$ Invest Dermatol. 2013;133 (6):1506-1511. doi:10.1038/jid.2012.472

49. Ingram JR, Piguet V. Phenotypic heterogeneity in hidradenitis suppurativa (acne inversa): classification is an essential step toward personalized therapy. J Invest Dermatol. 2013;133 (6): 1453-1456. doi:10.1038/jid.2012.476

50. Pink AE, Simpson MA, Desai N, et al. Mutations in the $\gamma$ secretase genes NCSTN, PSENEN, and PSEN1 underlie rare forms of hidradenitis suppurativa (acne inversa). J Invest Dermatol. 2012;132(10):2459-2461. doi:10.1038/jid.2012.162

51. Ingram JR, Wood M, John B, Butler R, Anstey AV. Absence of pathogenic $\gamma$-secretase mutations in a South Wales cohort of familial and sporadic hidradenitis suppurativa (acne inversa). $\mathrm{Br}$ J Dermatol. 2013;168(4):874-876. doi:10.1111/bjd.12048

52. Nomura Y, Nomura T, Suzuki S, et al. A novel NCSTN mutation alone may be insufficient for the development of familial hidradenitis suppurativa. J Dermatol Sci. 2014;74(2):180-182. doi:10.1016/j.jdermsci.2014.01.013

53. Garg A, Strunk A. Risk of Alzheimer's disease is not increased among patients with hidradenitis suppurativa: a retrospective population-based cohort analysis. J Am Acad Dermatol. 2017;77 (1):176-177. doi:10.1016/j.jaad.2017.02.055

54. Theut Riis P, Egeberg A, Gislason GH, Jemec GB. Patients with hidradenitis suppurativa have no increased risk of Alzheimer disease. Br J Dermatol. 2017;177(1):273-275. doi:10.1111/ bjd. 15064

55. Frew JW, Vekic DA, Woods J, Cains GD. A systematic review and critical evaluation of reported pathogenic sequence variants in hidradenitis suppurativa. Br J Dermatol. 2017;177(4):987-998. doi: $10.1111 /$ bjd.15441

56. Frew JW, Navrazhina K. In silico analysis of gamma-secretasecomplex mutations in hidradenitis suppurativa demonstrates disease-specific substrate recognition and cleavage alterations. Front Med. 2019;6:206. doi:10.3389/fmed.2019.00206

57. Li A, Peng Y, Taiclet LM, Tanzi RE. Analysis of hidradenitis suppurativa-linked mutations in four genes and the effects of PSEN1-P242LfsX11 on cytokine and chemokine expression in macrophages. Hum Mol Genet. 2019;28(7):1173-1182. doi:10.1093/hmg/ddy414

58. Esme P, Esme M, Caliskan E. Increased prevalence of family history of Alzheimer's disease in hidradenitis suppurativa: crosssectional analysis of 192 HS patients. Dermatol Ther. 2020;33(6): e14219. doi:10.1111/dth.14219

59. Nada EA, Muhammad EMS, Ahmed SFM, Tamam AMS, Abdelhamed A. Assessment of the effect of metabolic syndrome on the autophagy marker LC3 in psoriasis vulgaris patients: a cross-sectional study. Clin Cosmet Investig Dermatol. 2020;13:1005-1013. doi:10.2147/CCID.S284300

60. Carrascosa JM, Theng C, Thaçi D. Spotlight on topical long-term management of plaque psoriasis. Clin Cosmet Investig Dermatol. 2020;13:495-498. doi:10.2147/CCID.S254114

61. Fu Y, Lee $\mathrm{CH}$, Chi CC. Association of psoriasis with inflammatory bowel disease: a systematic review and meta-analysis. JAMA Dermatol. 2018;154(12):1417-1423. doi:10.1001/jamaderm atol.2018.3631

62. Soliman M. Patient-reported disease severity and quality of life among Arabic psoriatic patients: a cross-sectional survey. Clin Cosmet Investig Dermatol. 2020;13:601-609. doi:10.2147/CCID. S269909
63. Ezzedine K, Fougerousse AC, Aubert R, et al. Individual burden of psoriasis (I-BOP): building and validation of a new scoring tool for patients with psoriasis. Clin Cosmet Investig Dermatol. 2020;13:325-332. doi:10.2147/CCID.S249776

64. Orrell KA, Vakharia PP, Hagstrom EL, Brieva J, West DP, Nardone B. Prevalence of chronic hepatitis B and C in psoriasis patients: a cross-sectional study in a large US population. $J \mathrm{Am}$ Acad Dermatol. 2017;77(3):572-573. doi:10.1016/j.jaad.20 17.05.020

65. Leisner MZ, Riis JL, Schwartz S, Iversen L, Østergaard SD, Olsen MS. Psoriasis and risk of mental disorders in Denmark. JAMA Dermatol. 2019;155(6):745-747. doi:10.1001/jamad ermatol.2019.0039

66. Kim M, Park HE, Lee SH, Han K, Lee JH. Increased risk of Alzheimer's disease in patients with psoriasis: a nationwide population-based cohort study. Sci Rep. 2020;10(1):6454. doi:10.1038/ s41598-020-63550-2

67. Huang KL, Yeh CC, Wu SI, et al. Risk of dementia among individuals with psoriasis: a nationwide population-based cohort study in Taiwan. J Clin Psychiatry. 2019;80(3):18m12462. doi: $10.4088 / \mathrm{JCP} .18 \mathrm{~m} 12462$

68. Lin CC, Lin HC, Chiu HW. Association between psoriasis and dementia: a population-based case-control study. Am J Clin Dermatol. 2019;20(3):457-463. doi:10.1007/s40257-018-00420-8

69. Pezzolo E, Mutlu U, Vernooij MW, et al. Psoriasis is not associated with cognition, brain imaging markers and risk of dementia: the Rotterdam Study. J Am Acad Dermatol. 2018. doi:10.1016/j.jaad.2018.07.046

70. Liu L, Chen ST, Li HJ, et al. Association between psoriasis and dementia: current evidence. Front Aging Neurosci. 2020;12:570992. doi:10.3389/fnagi.2020.570992

71. Abuabara K, Azfar RS, Shin DB, Neimann AL, Troxel AB, Gelfand JM. Cause-specific mortality in patients with severe psoriasis: a population-based cohort study in the U.K. Br J Dermatol. 2010;163(3):586-592. doi:10.1111/j.1365-2133.2010.09941.x

72. Gisondi P, Sala F, Alessandrini F, et al. Mild cognitive impairment in patients with moderate to severe chronic plaque psoriasis. Dermatology. 2014;228(1):78-85. doi:10.1159/000357220

73. Colgecen E, Celikbilek A, Keskin DT. Cognitive impairment in patients with psoriasis: a cross-sectional study using the Montreal cognitive assessment. Am J Clin Dermatol. 2016;17(4):413-419. doi:10.1007/s40257-016-0187-3

74. Decourt B, Lahiri DK, Sabbagh MN. Targeting tumor necrosis factor alpha for Alzheimer's disease. Curr Alzheimer Res. 2017;14(4):412-425. doi:10.2174/1567205013666160930110551

75. Zhou M, Xu R, Kaelber DC, Gurney ME. Tumor necrosis factor (TNF) blocking agents are associated with lower risk for Alzheimer's disease in patients with rheumatoid arthritis and psoriasis. PLoS One. 2020;15(3):e0229819. doi:10.1371/journal. pone.0229819

76. Rich P, Bourcier M, Sofen H, et al. Ustekinumab improves nail disease in patients with moderate-to-severe psoriasis: results from PHOENIX 1. Br J Dermatol. 2014;170(2):398-407. doi:10.1111/ bjd. 12632

77. Mohammadi Shahrokhi V, Ravari A, Mirzaei T, Zare-Bidaki M, Asadikaram G, Arababadi MK. IL-17A and IL-23: plausible risk factors to induce age-associated inflammation in Alzheimer's disease. Immunol Invest. 2018;47(8):812-822. doi:10.1080/ 08820139.2018.1504300

78. Heppner FL, Ransohoff RM, Becher B. Immune attack: the role of inflammation in Alzheimer disease. Nat Rev Neurosci. 2015;16 (6):358-372. doi:10.1038/nrn3880

79. Vom Berg J, Prokop S, Miller KR, et al. Inhibition of IL-12/IL-23 signaling reduces Alzheimer's disease-like pathology and cognitive decline. Nat Med. 2012;18(12):1812-1819. doi:10.1038/ nm.2965 
80. Belloy ME, Napolioni V, Greicius MD. A quarter century of APOE and Alzheimer's disease: progress to date and the path forward. Neuron. 2019;101(5):820-838. doi:10.1016/j. neuron.2019.01.056

81. Al Harthi F, Huraib GB, Zouman A, Arfin M, Tariq M, AlAsmari A. Apolipoprotein E gene polymorphism and serum lipid profile in Saudi patients with psoriasis. Dis Markers. 2014;2014:239645. doi:10.1155/2014/239645

82. Campalani E, Allen MH, Fairhurst D, et al. Apolipoprotein E gene polymorphisms are associated with psoriasis but do not determine disease response to acitretin. Br J Dermatol. 2006;154 (2):345-352. doi:10.1111/j.1365-2133.2005.06950.x

83. Han Y, Liu T, Lu L. Apolipoprotein E gene polymorphism in psoriasis: a meta-analysis. Arch Med Res. 2013;44(1):46-53. doi:10.1016/j.arcmed.2012.10.009

84. Coto-Segura P, Coto E, Alvarez V, et al. Apolipoprotein $\varepsilon 4$ allele is associated with psoriasis severity. Arch Dermatol Res. 2010;302(2):145-149. doi:10.1007/s00403-009-1002-2

85. Yokoyama JS, Wang Y, Schork AJ, et al. Association between genetic traits for immune-mediated diseases and Alzheimer disease. JAMA Neurol. 2016;73(6):691-697. doi:10.1001/ jamaneurol.2016.0150

86. Ospina-Romero M, Glymour MM, Hayes-Larson E, et al. Association between Alzheimer disease and cancer with evaluation of study biases: a systematic review and meta-analysis. JAMA Network Open. 2020;3(11):e2025515. doi:10.1001/ jamanetworkopen.2020.25515

87. Roe CM, Fitzpatrick AL, Xiong C, et al. Cancer linked to Alzheimer disease but not vascular dementia. Neurology. 2010;74(2):106-112. doi:10.1212/WNL.0b013e3181c91873

88. Leiter U, Keim U, Garbe C. Epidemiology of skin cancer: update 2019. Adv Exp Med Biol. 2020;1268:123-139.

89. Schmidt SA, Ording AG, Horváth-Puhó E, Sørensen HT, Henderson VW. Non-melanoma skin cancer and risk of Alzheimer's disease and all-cause dementia. PLoS One. 2017;12 (2):e0171527. doi:10.1371/journal.pone.0171527

90. Ibler E, Tran G, Orrell KA, et al. Inverse association for diagnosis of Alzheimer's disease subsequent to both melanoma and nonmelanoma skin cancers in a large, urban, single-centre, Midwestern US patient population. J Eur Acad Dermatol Venereol. 2018;32(11):1893-1896. doi:10.1111/jdv.14952

91. Houck AL, Seddighi S, Driver JA. At the crossroads between neurodegeneration and cancer: a review of overlapping biology and its implications. Curr Aging Sci. 2018;11(2):77-89. doi:10.2174/1874609811666180223154436

92. Nakanishi A, Minami A, Kitagishi Y, Ogura Y, Matsuda S. BRCA1 and p53 tumor suppressor molecules in Alzheimer's disease. Int J Mol Sci. 2015;16(2):2879-2892. doi:10.3390/ ijms 16022879

93. Ma SL, Tang NL, Tam CW, et al. A PIN1 polymorphism that prevents its suppression by AP4 associates with delayed onset of Alzheimer's disease. Neurobiol Aging. 2012;33(4):804-813. doi:10.1016/j.neurobiolaging.2010.05.018

94. Segat L, Pontillo A, Annoni G, et al. PIN1 promoter polymorphisms are associated with Alzheimer's disease. Neurobiol Aging. 2007;28(1):69-74. doi:10.1016/j.neurobiolaging.2005.11.009
95. Kumar S, Reddy PH. A new discovery of MicroRNA-455-3p in Alzheimer's disease. J Alzheimers Dis. 2019;72(s1):S117-s130. doi:10.3233/JAD-190583

96. Galvão F, Grokoski KC, da Silva BB, Lamers ML, Siqueira IR. The amyloid precursor protein (APP) processing as a biological link between Alzheimer's disease and cancer. Ageing Res Rev. 2019;49:83-91. doi:10.1016/j.arr.2018.11.007

97. Botelho MG, Wang X, Arndt-Jovin DJ, Becker D, Jovin TM. Induction of terminal differentiation in melanoma cells on downregulation of $\beta$-amyloid precursor protein. $J$ Invest Dermatol. 2010;130(5):1400-1410. doi:10.1038/jid.2009.296

98. Matafora V, Farris F, Restuccia U, et al. Amyloid aggregates accumulate in melanoma metastasis modulating YAP activity. EMBO Rep. 2020;21(9):e50446. doi:10.15252/embr.202050446

99. Cummings J. What can be inferred from the interruption of the semagacestat trial for treatment of Alzheimer's disease? Biol Psychiatry. 2010;68(10):876-878. doi:10.1016/j.biopsych.20 10.09.020

100. $\mathrm{Li} \mathrm{T}$, Wen $\mathrm{H}$, Brayton $\mathrm{C}$, et al. Moderate reduction of gamma-secretase attenuates amyloid burden and limits mechanism-based liabilities. $J$ Neurosci. 2007;27 (40):10849-10859. doi:10.1523/JNEUROSCI.2152-07.2007

101. Ostendorf BN, Bilanovic J, Adaku N, et al. Common germline variants of the human APOE gene modulate melanoma progression and survival. Nat Med. 2020;26(7):1048-1053. doi:10.1038/ s41591-020-0879-3

102. Lövdén M, Xu W, Wang HX. Lifestyle change and the prevention of cognitive decline and dementia: what is the evidence? Curr Opin Psychiatry. 2013;26(3):239-243. doi:10.1097/ YCO.0b013e32835f4135

103. D'Orazio J, Jarrett S, Amaro-Ortiz A, Scott T. UV radiation and the skin. Int J Mol Sci. 2013;14(6):12222-12248. doi:10.3390/ ijms140612222

104. Steding-Jessen M, Birch-Johansen F, Jensen A, Schüz J, Kjær SK, Dalton SO. Socioeconomic status and non-melanoma skin cancer: a nationwide cohort study of incidence and survival in Denmark. Cancer Epidemiol. 2010;34(6):689-695. doi:10.1016/j.canep.201 0.06 .011

105. Tillement JP, Lecanu L, Papadopoulos V. Amyloidosis and neurodegenerative diseases: current treatments and new pharmacological options. Pharmacology. 2010;85(1):1-17. doi:10.1159/ 000259044

106. Schreml S, Kaiser E, Landthaler M, Szeimies RM, Babilas P. Amyloid in skin and brain: what's the link? Exp Dermatol. 2010;19(11):953-957. doi:10.1111/j.1600-0625.2010.01166.x

107. Awal G, Kaur S. Association of cutaneous amyloidosis with neurodegenerative amyloidosis: correlation or coincidence? J Clin Aesthet Dermatol. 2018;11(4):25-27.

108. Chen G, Chen ZM, Fan XY, et al. Gut-brain-skin axis in psoriasis: a review. Dermatol Ther. 2021;11(1):25-38. doi:10.1007/s13555020-00466-9

109. Neau JP, Godeneche G, Mathis S, Guillet G. Neurodermatology. Handb Clin Neurol. 2014;121:1561-1594. 


\section{Publish your work in this journal}

Clinical, Cosmetic and Investigational Dermatology is an international, peer-reviewed, open access, online journal that focuses on the latest clinical and experimental research in all aspects of skin disease and cosmetic interventions. This journal is indexed on CAS.
The manuscript management system is completely online and includes a very quick and fair peer-review system, which is all easy to use. Visit http://www.dovepress.com/testimonials.php to read real quotes from published authors.

Submit your manuscript here: https://www.dovepress.com/clinical-cosmetic-and-investigational-dermatology-journal 